\title{
Co-delivery of plasmid DNA and doxorubicin by solid lipid nanoparticles for lung cancer therapy
}

\author{
YIQUN HAN $^{1}$, PENG ZHANG ${ }^{1}$, YUANYUAN CHEN ${ }^{1}$, JIPING SUN $^{1}$ and FANSHENG KONG ${ }^{2}$ \\ Departments of ${ }^{1}$ Respiratory Medicine and ${ }^{2}$ Hematology, \\ General Hospital of Ji'nan Command of Chinese PLA, Ji'nan, Shandong 250031, P.R. China
}

Received November 30, 2013; Accepted April 8, 2014

DOI: $10.3892 / \mathrm{ijmm} .2014 .1770$

\begin{abstract}
The co-delivery of DNA and antitumor drugs has the potential to treat cancer. In this study, we aimed to develop surface-modified, co-encapsulated solid lipid nanoparticles (SLN) containing enhanced green fluorescence protein plasmid (pEGFP) and doxorubicin (DOX) in order to create a multifunctional delivery system that targets lung cancer cells, in an effort to improve the efficacy of cancer therapy. DOX- and pEGFPloaded SLN were prepared separately and then mixed to form co-encapsulated SLN (SLN/DE). Transferrin (Tf)-containing ligands were used for the surface coating of the vectors. The in vitro transfection efficiency of the modified vectors was evaluated using a human alveolar adenocarcinoma cell line (A549 cells) and the in vivo transfection efficiency of the modified vectors was evaluated using mice bearing A549 tumors. The Tf-modified DOX and pEGFP co-encapsulated SLN (T-SLN/ DE) had a particle size of $267 \mathrm{~nm}$ with a $42 \mathrm{mV}$ surface charge. The in vitro cytotoxicity of T-SLN/DE was low (cell viability was between 80 and $100 \%$ compared with the controls). T-SLN/ DE displayed a remarkable therapeutic effect both in drug delivery and gene therapy. In conclusion, our results demonstrate that the multifunctional delivery system can improve the efficacy of cancer therapy through the combination of gene therapy and chemotherapy. In addition, the coating of active targeting ligands can improve the efficacy of the carriers at targeting lung cancer cells. Thus, the novel gene and drug delivery system offers an effective strategy for lung cancer gene therapy.
\end{abstract}

\section{Introduction}

Lung cancer is the leading cause of cancer-related mortality worldwide, and the majority of patients present with metastatic stage IV disease at diagnosis $(1,2)$. The American Cancer

Correspondence to: Dr Fansheng Kong, Department of Hematology, General Hospital of Ji'nan Command of Chinese PLA, 25 Shifan Road, Ji'nan, Shandong 250031, P.R. China

E-mail: kongfanshengphd@hotmail.com kongfanshengphd@yahoo.com.cn

Key words: gene therapy, multifunctional delivery system, active targeting, co-delivery
Society estimated that 221,130 individuals in the United States developed lung cancer in 2011, with the number of deaths reaching 156,940 that year (3). Moreover, the relative 5-year survival ratio of patients with lung or bronchus cancer remains relatively low. Therefore, novel treatment strategies for lung cancer are urgently required (4).

Chemotherapeutics are the mainstream strategy for the treatment of localized and metastasized cancers. However, the development of multidrug resistance (MDR) of cancer cells, and the systemic toxic side-effects resulting from the unspecific localization of anticancer drugs to non-tumor areas are major obstacles to the success of chemotherapy in many types of cancer (5-7). A new combination therapeutic strategy for cancer, having the advantages of the co-delivery of more than one therapeutic agent in one delivery system, has recently been shown to be more effective than monotherapy by providing the potential synergistic effects of different treatment mechanisms. The co-delivery of nucleic acids and chemotherapeutics has been suggested to achieve the combined effect of gene therapy and chemotherapy (8-11). To date, attempts have been made to simultaneously deliver genes and drugs into cancer cells using liposomes (12), polymeric nanoparticles $(13,14)$, dendrimers (15), etc.

Our group has also worked on nanoparticulate drug/gene delivery systems (16-19). Solid lipid nanoparticles (SLN) have been widely developed in our laboratory as they have certain advantages, such as being less toxic, having low immunogenicity, and are easily modified $(16,17)$. They also offer a number of technological advantages, including better storage stability in comparison to liposomes, the possibility of steam sterilization and lyophilization, and large scale production with qualified production lines $(20,21)$.

Transferrin (Tf), an iron-binding glycoprotein, is a well-studied ligand for delivering anticancer drugs/genes due to the increased number of Tf receptors found in tumor cells as compared to normal cells (22). This receptor-mediated endocytosis may facilitate the delivery of drugs/genes into cells $(23,24)$. Polyethylene glycol (PEG)-phosphatidylethanolamine (PEG-PE) conjugates with various PEG lengths and terminaltargeted moieties can provide extremely stable, long-circulating, and actively targeted nanocarriers, which spontaneously accumulate at specific sites (25-27). In this study, Tf was linked to PEG-PE to form Tf-PEG-PE as ligands for the surface modification of nanocarriers. 
In the present study, Tf-PEG-PE was synthesized and modified onto the surface of the gene- and drug-loaded SLN. The in vivo transfection efficiency and antitumor effects of the novel modified vectors were evaluated using mice bearing A549 (human alveolar adenocarcinoma cell line) tumors. This system was expected to achieve stable drug and gene loading capacity, prolong the circulation time by PEG shielding, be recognized by the Tf receptor on A549 cells and internalized through receptor-mediated endocytosis, and finally, to achieve the co-delivery of both drug and gene therapeutic effects.

\section{Materials and methods}

Materials. Human Tf (iron-free), stearic acid, L- $\alpha$-phosphatidylethanolamine (PE), 3-(4,5-dimethylthiazol-2-yl)-2, 5-diphenyltetrazolium bromide (MTT), 2-iminothiolane (Traut's reagent), doxorubicin hydrochloride (DOX $\cdot \mathrm{HCl})$, and dimethyldioctadecylammonium bromide (DDAB), were purchased from Sigma-Aldrich Co., Ltd. (St. Louis, MO, USA). Injectable soya lecithin was obtained from Shanghai Taiwei Pharmaceutical Co., Ltd. (Shanghai, China). Maleimide$\mathrm{PEG}_{2000}-\mathrm{COOH}$ was purchased from Shanghai Yare Biotech, Inc. (Shanghai, China). The enhanced green fluorescence protein plasmid (pEGFP)-N1 was provided by Shandong University (Shandong, China). Quant-iT ${ }^{\mathrm{TM}}$ PicoGreen $^{\circledR}$ dsDNA quantification reagent was obtained from Invitrogen/Life Technologies (Carlsbad, CA, USA). A549 cells were obtained from the American Type Culture Collection (ATCC; Manassas, VA, USA). All other chemicals were of analytical grade or higher.

Animals. BALB/c mice (4-6 weeks old; weighing, 25-30 g) were purchased from the Medical Animal Test Center of Shandong Province and housed under standard laboratory conditions. All animal experiments complied with the requirements of the National Act on the Use of Experimental Animals (China).

Synthesis of Tf-PEG-PE. The Tf-PEG-PE ligands were synthesized as described in a previous study of ours (17). Briefly, maleimide- $\mathrm{PEG}_{2000}-\mathrm{COOH}$ was dissolved with dimethyl sulfoxide (DMSO) and stirred with PE as a mixture. 1-[3-(Dimethylamino)propyl]-3-ethylcarbodiimide hydrochloride $(\mathrm{EDC} \cdot \mathrm{HCl})$ and triethylamine (TEA) were dissolved in DMSO and added dropwise into the mixture in an ice bath, and stirred to produce maleimide-PEG-CO-NH-PE. Tf was firstly modified with Traut's reagent to complete the thiolation of $\mathrm{Tf}$. The thiolated $\mathrm{Tf}$ was then added to the maleimide$\mathrm{PEG}_{2000}-\mathrm{COOH}$ solution and the mixture was incubated at room temperature with gentle stirring. The product was dialyzed against Milli-Q water to form the Tf-PEG-PE solution. The mixture was centrifuged and then resuspended in phosphate-buffered saline (PBS, pH 7.4).

Preparation of SLN and SLN/DE. The SLN/pEGFP complexes were prepared as follows: blank SLN was prepared following the nanoprecipitation method (solvent displacement technique) as previouly described $(16,17)$. Stearic acid $(50 \mathrm{mg})$ and injectable soya lecithin $(30 \mathrm{mg})$ were accurately weighed and dissolved in $10 \mathrm{ml}$ acetone. The organic phase was added dropwise into the $0.5 \%$ DDAB solution being stirred at $600 \mathrm{rpm}$ at room temperature. When complete evaporation of the organic solvent occurred, the redundant stabilizers were separated by ultracentrifugation at $1,000 \times \mathrm{g}, 4^{\circ} \mathrm{C}$ for $20 \mathrm{~min}$. The pellet was vortexed and resuspended in Milli-Q water, washed 3 times, filtered through a $0.45-\mu \mathrm{m}$ membrane, and adjusted to $\mathrm{pH} 7.0$ with sodium hydroxide. The SLN/pEGFP complexes were prepared by incubating the SLN with pEGFP. Briefly, pEGFP was mixed with SLN by vortexing the particles with a $5 \mathrm{mg}$ / $\mathrm{ml}$ solution of pEGFP for $30 \mathrm{sec}$. The mixture was incubated for $30 \mathrm{~min}$ at room temperature to form SLN/pEGFP (Fig. 1).

The SLN/DOX complexes were prepared as follows: DOX.HCl was stirred with TEA in DMSO overnight to obtain the DOX base (28). DOX (5 mg) and stearic acid (50 mg) and injectable soya lecithin $(30 \mathrm{mg})$ were dissolved in $10 \mathrm{ml}$ warm ethanol. The resultant organic solution was rapidly dispersed into distilled water under mechanical stirring at $600 \mathrm{rpm}$ in a water bath at $70^{\circ} \mathrm{C}$ for $5 \mathrm{~min}$. The obtained solution was then cooled to room temperature to facilitate the formation of SLN/DOX. The SLN/pEGFP solution was then added dropwise into the SLN/DOX solution and stirred at $400 \mathrm{rpm}$ to obtain SLN/DE (Fig. 1).

Preparation of T-SLN/DE. The Tf-PEG-PE ligands were dissolved in $20 \mathrm{ml}$ of PBS. The solution was then added dropwise into $40 \mathrm{ml}$ of SLN/DE complexes and stirred at $400 \mathrm{rpm}$ at room temperature, leading to the immediate modification. Subsequently, free Tf-PEG-PE was removed from the modified SLN/DE by gel chromatography using a Sephadex ${ }^{\circledR}$ G-50 column (GE Healthcare Life Sciences, Piscataway, NJ, USA). The obtained complexes was resuspended in Milli-Q water and filtered through a membrane with $0.45 \mu \mathrm{m}$ pore size to obtain T-SLN/DE. The T-SLN was prepared by the same procedure using blank SLN without loading pEGFP and DOX (Fig. 1).

\section{Characterization of SLN, SLN/DE and T-SLN/DE}

Physical-chemical characteristics. The mean particle size, polydispersity index (PDI) and zeta potential of SLN, SLN/DE and T-SLN/DE were analyzed by photon correlation spectroscopy (PCS) using a Zetasizer 3000 instrument (Malvern Instruments, Malvern, UK). The average particle size was expressed as the volume mean diameter.

Gene loading capacity. The PicoGreen-fluorometry assay was used to quantify the amount of pEGFP carried by SLN/DE and T-SLN/DE. The concentration of pEGFP was determined by fluorescence, comparing with the supernatant from blank SLN. The amount of pEGFP loaded in the SLN was calculated according to the linear calibration curve of pEGFP as follows: gene loading quantity $(\%)=($ total amount of pEGFP - the amount of free pEGFP)/total amount of pEGFP x100.

Drug loading (DL) ability encapsulation efficiency (EE) assay. The DL and EE of the SLN/DE and T-SLN/DE were determined by a subtraction method. Briefly, $0.2 \mathrm{ml}$ of T-SLN/DE complexes solution was centrifuged through a filter (EMD Millipore, Billerica, MA, USA) with a molecular weight cut-off of $3 \mathrm{kDa}$. Free DOX could pass through the filter, but T-SLN/DE could not pass through the filter. Unincorporated DOX in the solution was quantified by determining the absorbance at $485 \mathrm{~nm}$ using a spectrophotometer, as previously described (29). DL and EE were calculated using the following equations: $\mathrm{DL}=[$ concentration of (total DOX - free DOX) $] \mathrm{x}$ 


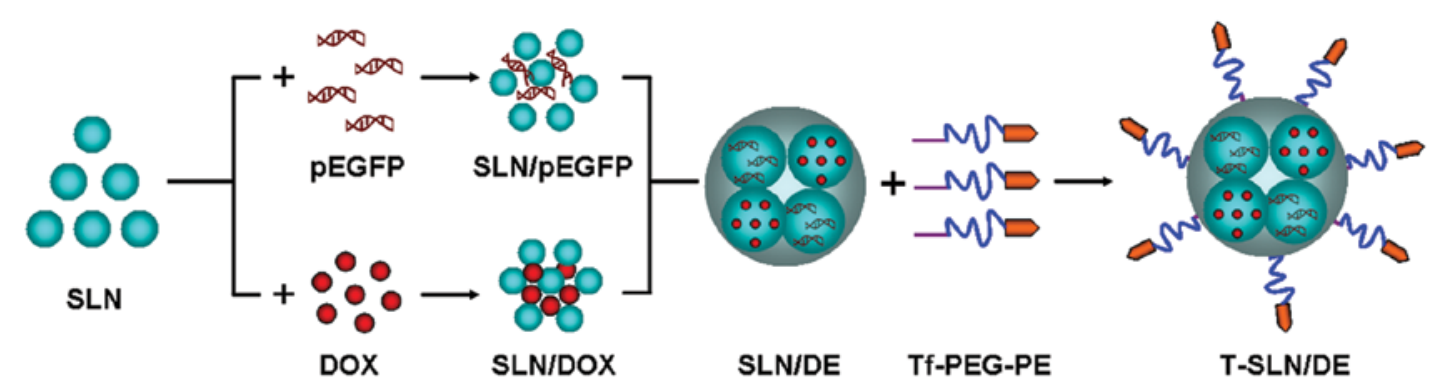

Figure 1. General scheme of the preparation of T-SLN/DE. SLN, solid lipid nanoparticles.

[concentration of (polymer + total DOX - free DOX) $]^{-1} \mathrm{x} 100 \%$ (Equation 1); EE = concentration of (total DOX - free DOX) $\mathrm{x}$ concentration of total $\mathrm{DOX}^{-1} \mathrm{x} 100 \%$ (Equation 2).

In vitro release assays. The in vitro gene release of SLN/ DE and T-SLN/DE was analyzed in PBS (pH 7.4). Typically, aliquots of complexes were suspended in $1 \mathrm{ml}$ of PBS (in Eppendorf $^{\circledR}$ tubes) and vortexed for $30 \mathrm{sec}$. The tubes were then placed in a $37^{\circ} \mathrm{C}$ shaking water bath $(100 \mathrm{rpm})$. Separate tubes were used for different data points. The suspensions were centrifuged at pre-determined time intevals $(1,000 \mathrm{x} \mathrm{g}$ for $30 \mathrm{~min}$ ) and the amount of pEGFP released into the supernatant was analyzed using the PicoGreen assay mentioned above. Background readings were obtained using the supernatants from the blank SLN.

The in vitro drug release of SLN/DE and T-SLN/DE was analyzed using a modified dialysis method $(30,31)$. Briefly, $0.5 \mathrm{ml}$ complexes were transferred into a dialysis membrane (MWCO 3000), and $0.5 \mathrm{ml}$ of free DOX solution in water $(0.25 \mathrm{mg} / \mathrm{ml})$ was used as the control. The solutions were then dialyzed against $20 \mathrm{ml}$ acetic acid sodium buffer at $\mathrm{pH} 5.5$ and PBS, both containing Tween-20 (0.5\%), at $37^{\circ} \mathrm{C}$ with gentle shaking. A total of $20 \mathrm{ml}$ of the surrounding dialysis medium was removed at pre-determined time points for analysis, and $20 \mathrm{ml}$ of fresh buffer at the relevant $\mathrm{pH}$ were added to the dialysis medium. The released DOX from the vectors was able to infiltrate through the dialysis bag as the molecular weight of the DOX was $<3,000$. The released DOX was quantified by determining the absorbance at $485 \mathrm{~nm}$ using a spectrophotometer (F-2500; Hitachi, Tokyo, Japan).

In vitro cytotoxicity evaluation. To examine the cytotoxicity, A549 cells were seeded in 48 -well plates at $1 \times 10^{5}$ cells/well and incubated for $24 \mathrm{~h}$ to allow cell attachment. The cells were incubated with SLN and T-SLN complexes at various concentrations $(10,20,50,100$ and $200 \mu \mathrm{g} / \mathrm{ml})$ for $48 \mathrm{~h}$ at $37^{\circ} \mathrm{C}$ and a $5 \% \mathrm{CO}_{2}$ atmosphere. The cells without incubation were used as the negative controls. Cell viability was assessed by MTT assay according to the manufacturer's instructions and the absorbance was measured at $570 \mathrm{~nm}$ using a microplate reader (Model 680; Bio-Rad, Hercules, CA, USA). Cells without the addition of MTT reagents were used as the blank to calibrate the spectrophotometer to zero absorbance. The relative cell viability (\%) was calculated as $\left(\mathrm{Abs}_{\text {sample }}-\mathrm{Abs}_{\text {blank }}\right) /\left(\mathrm{Abs}_{\text {control }}-\mathrm{Abs}_{\text {blank }}\right) \times 100$.

In vitro transfection analysis. For transfection efficiency analysis, the A549 cells were seeded into 24-well plates at a density of $1 \times 10^{4}$ cells/well and transfected the following day at $80-90 \%$ confluency. Prior to transfection, the media were replaced with $500 \mu \mathrm{l}$ transfection media containing T-SLN/DE. Naked pEGFP, blank SLN and SLN/DE were used as controls. The original incubation medium was replaced with $1 \mathrm{ml}$ of complete medium following incubation at $37^{\circ} \mathrm{C}$ for $4 \mathrm{~h}$ under a $5 \% \mathrm{CO}_{2}$ atmosphere. The cells were incubated and examined until $72 \mathrm{~h}$ post-transfection. To quantify the transfection efficiency, the cells were washed with $1 \mathrm{ml}$ of PBS $\left(100 \mathrm{~g}, 4^{\circ} \mathrm{C}\right.$ for $5 \mathrm{~min}$ ) and were detached with trypsin/EDTA. The supernatant was discarded and resuspended with $300 \mu \mathrm{l}$ of PBS, mixed well and subjected to flow cytometry to determine the amount of A549 cells which has been successfully transfected.

In vivo antitumor effects. Tumor-bearing mice were prepared by inoculating (subcutaneously) a suspension of A549 cells (1x $10^{6}$ cells) into the right armpit of BALB/c mice (32). Briefly, the mice were acclimatized at a temperature of $25 \pm 2^{\circ} \mathrm{C}$ and a relative humidity of $70 \pm 5 \%$ under natural light/dark conditions for 1 week before dosing. The mice were then subcutaneously injected in the right armpit with A549 cells suspended in PBS. Tumors were allowed to reach $4-5 \mathrm{~mm}$ in diameter before initiation of the experiments.

The in vivo anticancer activity of T-SLN/DE was evaluated against A549 solid tumors in mice. Five groups of tumor-bearing mice (6 mice per group) were used. The mice were injected with $10 \mathrm{mg} / \mathrm{kg}$ of T-SLN/DE, SLN/DE, SLN, free DOX solution, and $0.9 \%$ sodium chloride solution (blank control). All drugs were diluted with $0.9 \%$ sodium chloride $(100 \mu \mathrm{l})$, and all were administered through direct intratumoral injection. Following drug administration, mortality was monitored daily and tumor growth was determined by caliper measurement every 3 days. The tumor volume was calculated as follows, according to a previously described method (33): tumor volume $\left(\mathrm{mm}^{3}\right)=\left(\right.$ length $\mathrm{x}$ width $\left.{ }^{2}\right) / 2($ Equation 3$)$.

Statistical analysis. All experiments were repeated 3 times and all measurements were carried out in triplicate. The results are reported as the means \pm standard deviation (SD). Statistical significance was analyzed using the Student's t-test. The differences between experimental groups was considered significant when the $\mathrm{P}$-value was $<0.05(\mathrm{P}<0.05)$.

\section{Results}

Characterization of $T-S L N / D E$. The mean particle size, PDI, zeta potential, gene loading ability, DL and EE of SLN, 
Table I. Particle size, zeta potential and gene loading quantity of the different vectors.

\begin{tabular}{lccc}
\hline Sample characteristics & SLN & SLN/DE & T-SLN/DE \\
\hline Mean particle size (nm) & $82.1 \pm 2.9$ & $245.5 \pm 3.2$ & $286.5 \pm 3.9$ \\
Polydispersity index (PDI) & $0.13 \pm 0.03$ & $0.15 \pm 0.03$ & $0.14 \pm 0.02$ \\
Zeta potential (mV) & $+41.5 \pm 2.6$ & $+28.3 \pm 2.3$ & $+19.1 \pm 1.8$ \\
Gene loading quantity (\%) & N/A & 82 & 81 \\
DL $(\%)$ & N/A & $8.9 \pm 0.8$ & $8.7 \pm 0.9$ \\
EE $(\%)$ & N/A & $82.6 \pm 1.5$ & $81.9 \pm 1.3$ \\
\hline
\end{tabular}

SLN, solid lipid nanoparticles.

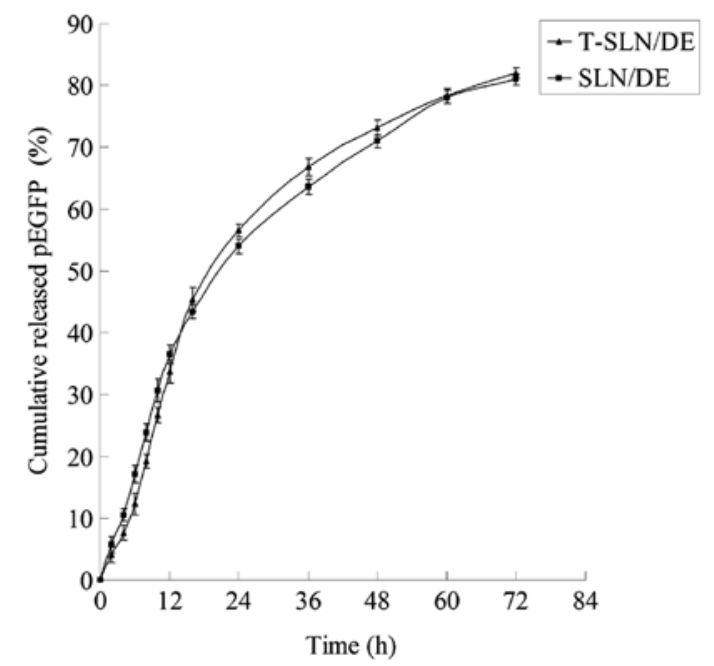

Figure 2. The in vitro gene release profiles of T-SLN/DE and SLN/DE. SLN, solid lipid nanoparticles.

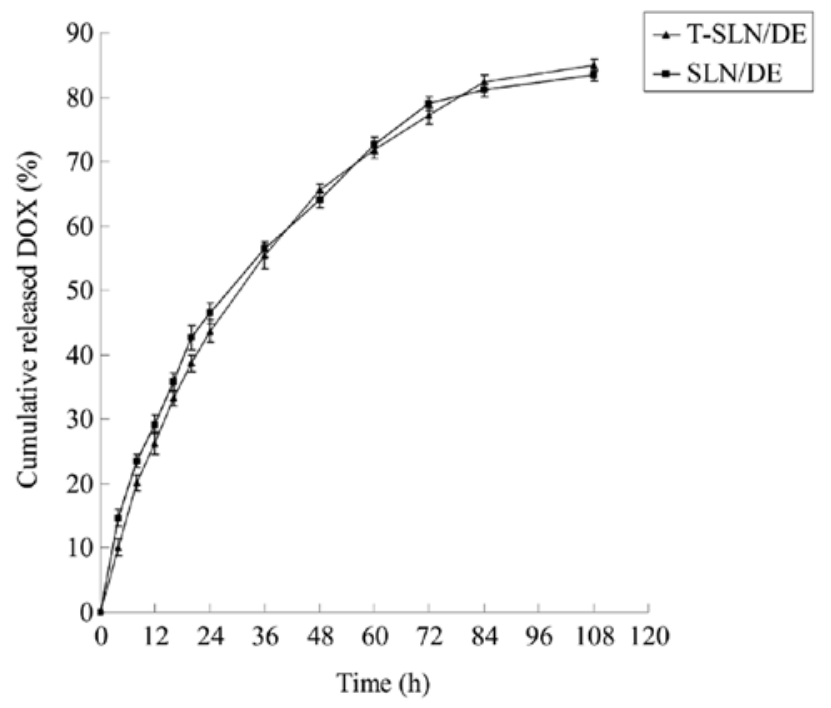

Figure 3. The in vitro drug release profiles of T-SLN/DE and SLN/DE. SLN, solid lipid nanoparticles.

SLN/DE and T-SLN/DE were characterized and are summarized in Table I.

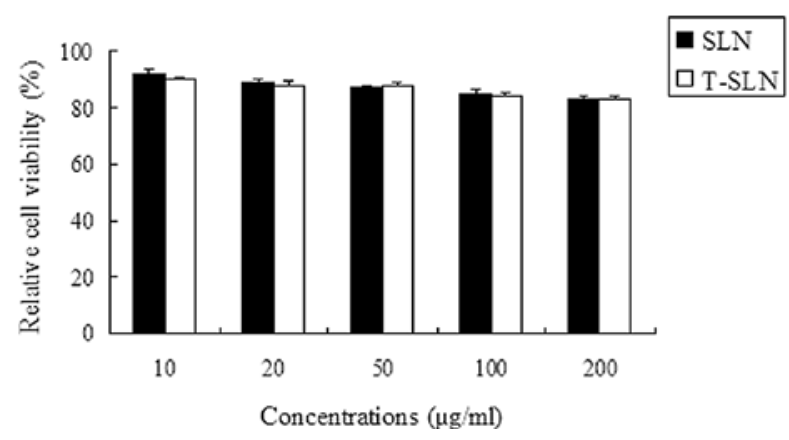

Figure 4. In vitro viability of A549 cells transfected with T-SLN and SLN SLN, solid lipid nanoparticles.

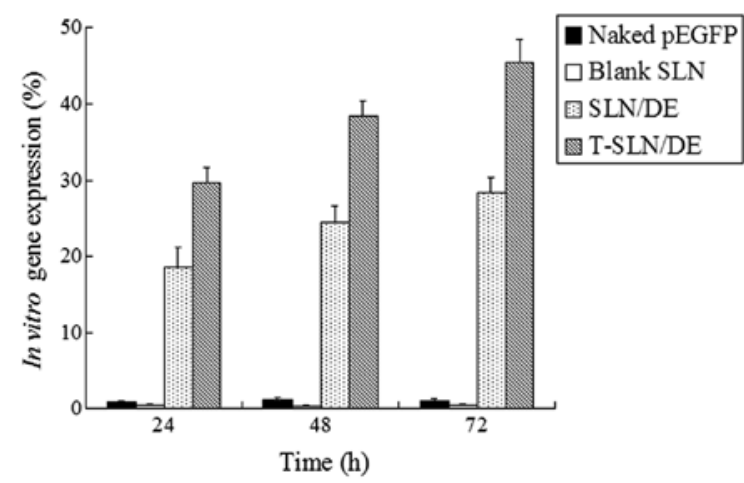

Figure 5. In vitro transfection efficiencies of the different systems in A549 cells.

In vitro release assays. The in vitro gene and drug release profiles of T-SLN/DE and SLN/DE are illustrated in Figs. 2 and 3. The gene and drug release of T-SLN/DE and SLN/DE reached over $80 \%$ at the time point of $72 \mathrm{~h}$.

In vitro cytotoxicity evaluation. The in vitro cytotoxicity of T-SLN and SLN at various concentrations was evaluated by MTT assay in the A549 cells. As illustrated in Fig. 4, the cell viability of the A549 cells transfected with the vectors at the examined concentration range $(10-200 \mu \mathrm{g} / \mathrm{ml})$ was above $80 \%$ compared with the controls (cells without incubation).

In vitro transfection analysis. The in vitro transfection efficiencies of T-SLN/DE and SLN/DE were evaluated in the A549 cells until $72 \mathrm{~h}$ of transfection (Fig. 5). T-SLN/DE showed a higher transfection efficiency than SLN/DE and naked pEGFP at 48 and $72 \mathrm{~h}$ post-transfection $(\mathrm{P}<0.05)$.

In vivo anticancer efficacy. The in vivo antitumor efficiency of T-SLN/DE and SLN/DE was observed against A549 solid tumors in mice. The tumor growth curves of each group are presented in Fig. 6. The results indicated that treatment with SLN/DE resulted in smaller tumor volume compared with free DOX; treatment with T-SLN/DE resulted in a smaller tumor volume compared with the vectors. These results illustrate that the encapsulation of DOX in SLN enhances the anticancer activity of DOX in vivo; Tf-modified SLN/DE exerts more enhanced antitumor effects compared with the vectors not modified with Tf. 


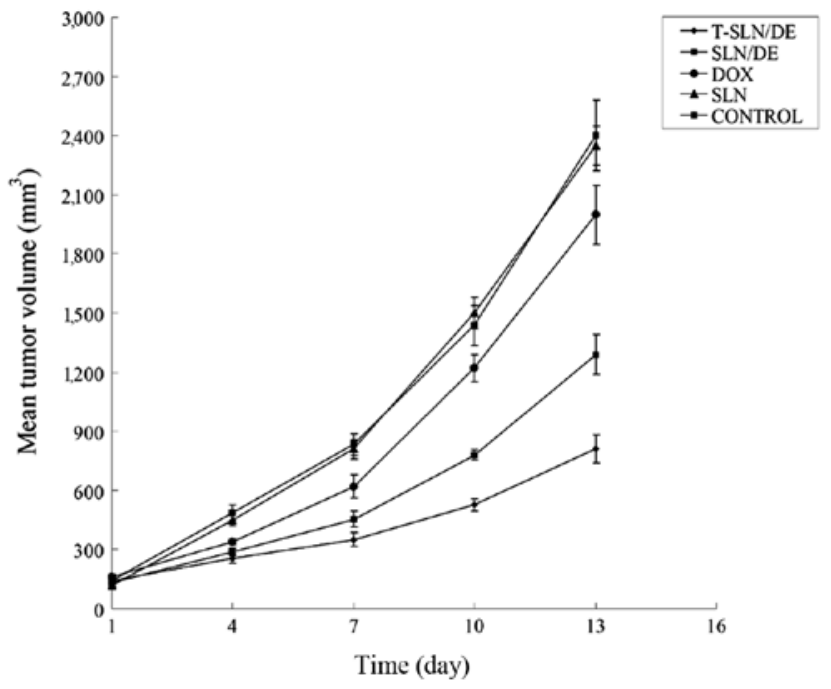

Figure 6. The tumor growth curves of T-SLN/DE and SLN/DE SLN, solid lipid nanoparticles.

\section{Discussion}

SLN have been previously developed by our group for anticancer drug/gene delivery therapy (16-19). This has a number of technological advantages, including rapid uptake by cells, protection of the incorporated compound against chemical degradation and potential for large-scale production $(34,35)$. This study aimd to develop surface-modified, co-encapsulated SLN containing pEGFP and DOX in order to create a multifunctional delivery system that will target lung cancer cells and increase the therapeutic efficacy.

To overcome the barriers of the cellular membrane and achieve efficient gene therapy, Tf-PEG-PE was applied as a modifier that was coated on the nanoparticle surface after the preparation of the gene- and drug-loaded cationic SLN (SLN/DE). Tf is an iron-binding glycoprotein, which is particularly useful in targeting cancer cells, as many cancer cells overexpress $\mathrm{Tf}$ receptor (TfR) on their surface $(22,23,36)$. In this study, T-SLN/DE had a size of $287 \mathrm{~nm}$ and a zeta potential of $+19 \mathrm{mV}$ (Table I).

PicoGreen-fluorometry assay was carried out to determine the binding ability and in vitro gene release of T-SLN/DE and SLN/DE. The gene loading efficiency of T-SLN/DE was $81 \%$, which did not differ significantly from that of SLN/pEGFP (82\%) (Table I). The results proved that the binding of the Tf-PEG-PE ligand did not detach the pEGFP from the complexes. The in vitro release profile of T-SLN/DE had almost the same behavior with SLN/DE (Fig. 2). During the first $12 \mathrm{~h}, \mathrm{~T}-\mathrm{SLN} / \mathrm{DE}$ showed a slightly slower release activity than SLN/DE. This phenomenon may be due to the surface coating of ligands initially hindering the release of pEGFP. After $12 \mathrm{~h}$ and until the end of the release analysis, the total amount of pEGFP delivered from the 2 types of vehicles was almost the same (over 80\%). The DL and EE of T-SLN/DE and SLN/DE were determined by a subtraction method. The DL of SLN/DE and T-SLN/DE were approximately $9 \%$ and the EE of both vectors was approximately $82 \%$ (Table I). The results demonstrated that the binding of the Tf-PEG-PE ligand did not detach the DOX from the complexes and that the modi- fied vectors were stable. The in vitro drug release profile of T-SLN/DE a showed slightly slower release than that of SLN/ DE during the first $24 \mathrm{~h}$ (Fig. 3). At the end of the release analysis, the total amount of drugs delivered from the 2 types of vehicles was almost the same.

In vitro cytotoxicity and transfection analyses were carried out using A549 cells. The viability of the cells transfected with T-SLN and SLN at the examined concentration range was over $80 \%$ compared with the controls (Fig. 4). T-SLN did not show a higher cytotoxicity than SLN at all concentrations. In comparison to naked $\mathrm{pEGFP}$ and SLN/DE, T-SLN/DE had a greater transfection efficiency at 48 and $72 \mathrm{~h}(\mathrm{P}<0.05)$ (Fig. 5). This may be explained by the receptor-mediated active targeting mechanism: Tf on the SLN/DE surface was more likely to bind to the A549 cells through the TfR on the cells and deliver the gene more easily into the cells.

The antitumor efficacy of T-SLN/DE was further examined in tumor-bearing mice. The mice were injected with $10 \mathrm{mg} /$ $\mathrm{kg}$ of T-SLN/DE, SLN/DE, SLN, free DOX solution into the tumor site; $0.9 \%$ sodium chloride solution was used as the blank control. The tumor growth rate was not found to significantly decrease with free DOX treatment and SLN (similar results were observed with $0.9 \%$ sodium chloride solution). The tumor growth rate was significantly decreased in the group treated with SLN/DE. Furthermore, T-SLN/DE showed a greater antitumor effect than the unmodified SLN/DE in vivo. These results indicate that Tf-modified drug- and gene-loaded SLN have improved antitumor effects and an excellent gene transfection efficiency. Therefore, T-SLN/DE can be used as a promising vehicle for the delivery of antitumor drugs and genes and may significantly contribute to cancer therapy.

In conclusion, in the current study, Tf-modified co-encapsulated DOX- and pEGFP-loaded SLN were prepared and characterized according to their size, loading efficiency and in vitro drug release. The results revealed that T-SLN/DE may significantly improve the gene transfection efficiency of the vector and successfully control the tumor growth rate in tumor-bearing mice. Conclusively, Tf may function as an excellent active targeting ligand to improve the cell targeting ability of carriers. Furthermore, the modified co-delivery system may function comprehensively to improve the efficacy of anticancer therapy. Our data indicate that this system may be an excellent carrier for the delivery of both plasmid DNA and DOX, leading to the enhanced efficacy of antitumor therapy.

\section{References}

1. Shepherd FA, Bunn PA and Paz-Ares L: Lung cancer in 2013: state of the art therapy for metastatic disease. Am Soc Clin Oncol Educ Book: 339-346, 2013.

2. Sundaram S, Trivedi R, Durairaj C, Ramesh R, Ambati BK and Kompella UB: Targeted drug and gene delivery systems for lung cancer therapy. Clin Cancer Res 15: 7299-7308, 2009.

3. Siegel R, Ward E, Brawley O and Jamel A: Cancer statistics, 2011: the impact of eliminating socioeconomic and racial disparities on premature cancer deaths. CA Cancer J Clin 61: 212-236, 2011.

4. Kawabata A, Baoum A, Ohta N, Jacquez S, Seo GM, Berkland C and Tamura M: Intratracheal administration of a nanoparticle-based therapy with the angiotensin II type 2 receptor gene attenuates lung cancer growth. Cancer Res 72: 2057-2067, 2012.

5. Bajelan E, Haeri A, Vali AM, Ostad SN and Dadashzadeh S: Co-delivery of doxorubicin and PSC 833 (Valspodar) by stealth nanoliposomes for efficient overcoming of multidrug resistance. J Pharm Pharm Sci 15: 568-582, 2012. 
6. Brannon-Peppas L and Blanchette JO: Nanoparticle and targeted systems for cancer therapy. Adv Drug Deliv Rev 56: 1649-1659, 2004.

7. Gottesman MM, Fojo $\mathrm{T}$ and Bates SE: Multidrug resistance in cancer: role of ATP-dependent transporters. Nat Rev Cancer 2: 48-58, 2002.

8. Liu C, Liu F, Feng L, Li M, Zhang J and Zhang N: The targeted co-delivery of DNA and doxorubicin to tumor cells via multifunctional PEI-PEG based nanoparticles. Biomaterials 34 2547-2564, 2013.

9. Zheng C, Zheng M, Gong P, Deng J, Yi H, Zhang P, Zhang Y, Liu P, Ma Y and Cai L: Polypeptide cationic micelles mediated co-delivery of docetaxel and siRNA for synergistic tumor therapy. Biomaterials 34: 3431-3438, 2013.

10. Loh XJ, Ong SJ, Tung YT and Choo HT: Co-delivery of drug and DNA from cationic dual-responsive micelles derived from poly(DMAEMA-co-PPGMA). Mater Sci Eng C Mater Biol Appl 33: 4545-4550, 2013.

11. Zhao J, Mi Y and Feng SS: Targeted co-delivery of docetaxel and siPlk1 by herceptin-conjugated vitamin E TPGS based immunomicelles. Biomaterials 34: 3411-3421, 2013.

12. Shim G, Han SE, Yu YH, et al: Trilysinoyl oleylamide-based cationic liposomes for systemic co-delivery of siRNA and an anticancer drug. J Control Release 155: 60-66, 2011.

13. Xu Z, Zhang Z, Chen Y, Chen L, Lin L and Li Y: The characteristics and performance of a multifunctional nanoassembly system for the co-delivery of docetaxel and iSur-pDNA in a mouse hepatocellular carcinoma model. Biomaterials 31: 916-922, 2010.

14. Qiu B, Ji M, Song X, et al: Co-delivery of docetaxel and endostatin by a biodegradable nanoparticle for the synergistic treatment of cervical cancer. Nanoscale Res Lett 7: 666, 2012.

15. Kaneshiro TL and Lu ZR: Targeted intracellular codelivery of chemotherapeutics and nucleic acid with a well-defined dendrimer-based nanoglobular carrier. Biomaterials 30 5660-5666, 2009.

16. Jiang Z, Sun C, Yin Z, Zhou F, Ge L, Liu X and Kong F: Comparison of two kinds of nanomedicine for targeted gene therapy: premodified or postmodified gene delivery systems. Int J Nanomedicine 7: 2019-2031, 2012.

17. Wang W, Zhou F, Ge L, Liu X and Kong F: Transferrin-PEG-PE modified dexamethasone conjugated cationic lipid carrier mediated gene delivery system for tumor-targeted transfection. Int J Nanomedicine 7: 2513-2522, 2012.

18. Kong F, Ge L, Liu X, Huang N and Zhou F: Mannanmodified PLGA nanoparticles for targeted gene delivery. Int J Photoenergy 2012: 926754, 2012.

19. Kong F, Zhou F, Ge L, Liu X and Wang Y: Mannosylated liposomes for targeted gene delivery. Int $\mathrm{J}$ Nanomedicine 7 : 1079-1089, 2012

20. Olbrich C, Bakowsky U, Lehr CM, Müller RH and Kneuer C: Cationic solid-lipid nanoparticles can efficiently bind and transfect plasmid DNA. J Control Release 77: 345-355, 2011.
21. Vighi E, Ruozi B, Montanari M, Battini R and Leo E Re-dispersible cationic solid lipid nanoparticles (SLNs) freeze-dried without cryoprotectors: characterization and ability to bind the pEGFP-plasmid. Eur J Pharm Biopharm 67: 320-328, 2007.

22. Bellocq NC, Pun SH, Jensen GS and Davis ME: Transferrin-containing, cyclodextrin polymer-based particles for tumor-targeted gene delivery. Bioconjug Chem 14: 1122-1132, 2003.

23. Li H and Qian ZM: Transferrin/transferrin receptor-mediated drug delivery. Med Res Rev 22: 225-250, 2002.

24. Maruyama K: Intracellular targeting delivery of liposomal drugs to solid tumors based on EPR effects. Adv Drug Deliv Rev 63: 161-169, 2011

25. Torchilin VP: Multifunctional nanocarriers. Adv Drug Deliv Rev 58: 1532-1555, 2006.

26. Torchilin VP: Recent advances with liposomes as pharmaceutical carriers. Nat Rev Drug Discov 4: 145-160, 2005.

27. Lukyanov AN, Gao Z, Mazzola L and Torchilin VP: Polyethylene glycol-diacyllipid micelles demonstrate increased acculumation in subcutaneous tumors in mice. Pharm Res 19: 1424-1429, 2002.

28. Miao J, Du YZ, Yuan H, Zhang XG and Hu FQ: Drug resistance reversal activity of anticancer drug loaded solid lipid nanoparticles in multi-drug resistant cancer cells. Colloids Surf B Biointerfaces 110: 74-80, 2013.

29. Gao X, Wang B, Wei X, et al: Preparation, characterization and application of star-shaped PCL/PEG micelles for the delivery of doxorubicin in the treatment of colon cancer. Int J Nanomedicine 8: 971-982, 2013.

30. Men K, Liu W, Li L, et al: Delivering instilled hydrophobic drug to the bladder by a cationic nanoparticle and thermo-sensitive hydrogel composite system. Nanoscale 4: 6425-6433, 2012.

31. Wang BL, Gao X, Men K, et al: Treating acute cystitis with biodegradable micelle-encapsulated quercetin. Int J Nanomedicine 7: 2239-2247, 2012

32. Li P,Liu D, Miao L, Liu C, Sun X, Liu Y and Zhang N: A pH-sensitive multifunctional gene carrier assembled via layer-by-layer technique for efficient gene delivery. Int J Nanomedicine 7: 925-939, 2012

33. Jia Y, Yuan M, Yuan $\mathrm{H}$, et al: Co-encapsulation of magnetic $\mathrm{Fe}_{3} \mathrm{O}_{4}$ nanoparticles and doxorubicin into biodegradable PLGA nanocarriers for intratumoral drug delivery. Int J Nanomedicine 7: 1697-1708, 2012

34. He SN, Li YL, Yan JJ, Zhang W, Du YZ, Yu HY, Hu FQ and Yuan H: Ternary nanoparticles composed of cationic solid lipid nanoparticles, protamine, and DNA for gene delivery. Int J Nanomedicine 8: 2859-2869, 2013

35. Mehnert W and Mäder K: Solid lipid nanoparticles: production, characterization and applications. Adv Drug Deliv Rev 47: 165-196, 2001.

36. Singh M: Transferrin as a targeting ligand for liposomes and anticancer drugs. Curr Pharm Des 5: 443-451, 1999. 